\title{
Comparative Study on Structural and Electrical Properties of Network and Continuous $\mathrm{Ni}_{80} \mathrm{Fe}_{20}$ Films
}

\author{
Jun-Sheng MA ${ }^{a}$, Ming-Peng $\mathrm{YU}^{\mathrm{b}}$, Kang $\mathrm{HE}^{\mathrm{c}}$ and Hong QIU ${ }^{\mathrm{d},{ }^{*}}$ \\ Department of Physics, School of Mathematics and Physics, University of Science and Technology \\ Beijing, 30 Xueyuanlu, Haidian District, Beijing 100083, China \\ amjs1209@126.com, byum@ustb.edu.cn, cb20150328@xs.ustb.edu.cn, 'diuhong@sas.ustb.edu.cn \\ ${ }^{*}$ Corresponding author
}

Keywords: $\mathrm{Ni}_{80} \mathrm{Fe}_{20}$ film, Network structure, Electrical property.

\begin{abstract}
Ni}_{80} \mathrm{Fe}_{20}$ films were deposited on anodic aluminium oxide (AAO) and $\mathrm{SiO}_{2} / \mathrm{Si}(100)$ substrates at $300 \mathrm{~K}$ by a magnetron sputtering system with the oblique target. The film grown on the AAO substrate has a network structure while that deposited on the $\mathrm{SiO}_{2} / \mathrm{Si}(100)$ substrate is continuous. The network film is formed by columnar granule connection. The columnar granule consists of many fine granular grains. The continuous film grows with thin columnar grains. The columnar grain size of the continuous film is almost equal to the granular grain size of the network film. A temperature dependence of the resistance within 3-300 K reveals that the network film exhibits a minimal resistance at about $35 \mathrm{~K}$. A logarithmic temperature dependence of conductance is verified at temperatures below $35 \mathrm{~K}$. The temperature coefficient of resistance of the network film is smaller than that of the continuous film in 150-300 K.
\end{abstract}

\section{Introduction}

Network NiFe films have been actively investigated by many research groups for scientific interesting and potential applications in magnetic recording devices [1-3]. Weston et al [1] studied a magnetic property of the network $\mathrm{Ni}_{81} \mathrm{Fe}_{19}$ films as a function of the film thickness. They found that the $20 \mathrm{~nm}$-thick network film had the highest coercivity. Tanaka et al [2] reported that a magnetic property of the $\mathrm{Ni}_{81} \mathrm{Fe}_{19}$ honeycomb network film was strongly governed by magnetic interactions at the network vertexes. Sun et al [3] found that the $\mathrm{CoO} / \mathrm{NiFe}$ bilayer network films exhibited large exchange bias and coercivity compared to the continuous bilayer films with the same layer thickness. Previous studies focused mainly on the magnetic properties of the network NiFe films. On the other hand, the metal particles connect with each other forming the electrically conducting network in the insulating atmosphere. As it is well known, the conducting mechanism in the conductive network can be explained by the percolation theory and is different from that of the continuous conductive films. Furthermore, the network film has a large specific surface area compared to the continuous film. The large specific surface area can influence its electrical property. Therefore, electrical properties of metal network films are significant for fundamental and practical viewpoints. Aprili et al [4,5] found that network $\mathrm{Ni}$ films grown on $\mathrm{SiO}_{2}$ had an anomaly conductance decrease with temperature at temperatures below $4 \mathrm{~K}$. They proposed that the anomaly conductance decrease was attributed to the Coulomb electron-electron interactions [5]. Recently, Fe and Ni network films have been grown on AAO substrates by sputtering. A temperature dependence of the resistance within 1.5-200 K revealed that the network films exhibited a minimal resistance at 30-40 K [6,7]. In the present work, $\mathrm{Ni}_{80} \mathrm{Fe}_{20}$ films are deposited on $\mathrm{AAO}$ and $\mathrm{SiO}_{2} / \mathrm{Si}(100)$ substrates by sputtering. The film structure is studied by using field emission scanning electron microscopy (FE-SEM). A temperature dependence of the resistance is measured in 3-300 $\mathrm{K}$ to investigate the electrical transport property in the films. This work further enriches and reproduces the results reported by references 6 and 7 . 


\section{Experimental Procedure}

The direct current magnetron sputtering system, which has the target inclined to the substrate at an angle of $45^{\circ}$, has been described elsewhere in detail [8]. The commercial AAO substrate (Whatman) has an average pore diameter of $100 \mathrm{~nm}$. The average pore wall width on the substrate surface is 25 $\mathrm{nm}$. The $\mathrm{SiO}_{2} / \mathrm{Si}(100)$ substrate is a $400 \mathrm{~nm}$-thick $\mathrm{SiO}_{2}$ layer prepared by thermal oxidation of the $\mathrm{Si}(100)$ surface. The $\mathrm{SiO}_{2} / \mathrm{Si}(100)$ substrates were ultrasonically rinsed in acetone, in deionized water and in ethanol, respectively. 200 nm-thick $\mathrm{Ni}_{80} \mathrm{Fe}_{20}$ films were sputter-deposited on AAO and $\mathrm{SiO}_{2} / \mathrm{Si}(100)$ substrates at $300 \mathrm{~K}$ using $\mathrm{Ni}_{80} \mathrm{Fe}_{20}$ target (99.99\% purity) of $50 \mathrm{~mm}$ in diameter. Prior to deposition, the chamber was evacuated to a pressure of $3 \times 10^{-4}$ Pa using a turbo molecular pump. A distance between the target and the substrate was $10 \mathrm{~cm}$. During the sputter-deposition, an Ar gas (99.9995\% purity) pressure was adjusted to $2.0 \mathrm{~Pa}$ and a sputtering power was fixed at $100 \mathrm{~W}$. The average deposition rate was $10 \mathrm{~nm} / \mathrm{min}$. The substrate holder was rotated by using a stepping motor during deposition in order to obtain the uniformly thick film. FE-SEM of SUPRA 55 (Zeiss) was used to observe the structure of the films. A resistance of the films was measured in the temperature range of $3 \mathrm{~K}$ to $300 \mathrm{~K}$ using the Cryogen-Magnet system of CFM-5T-H3-CFVTI-1.6K-24.5 (Cryogenic).

\section{Results and Discussion}
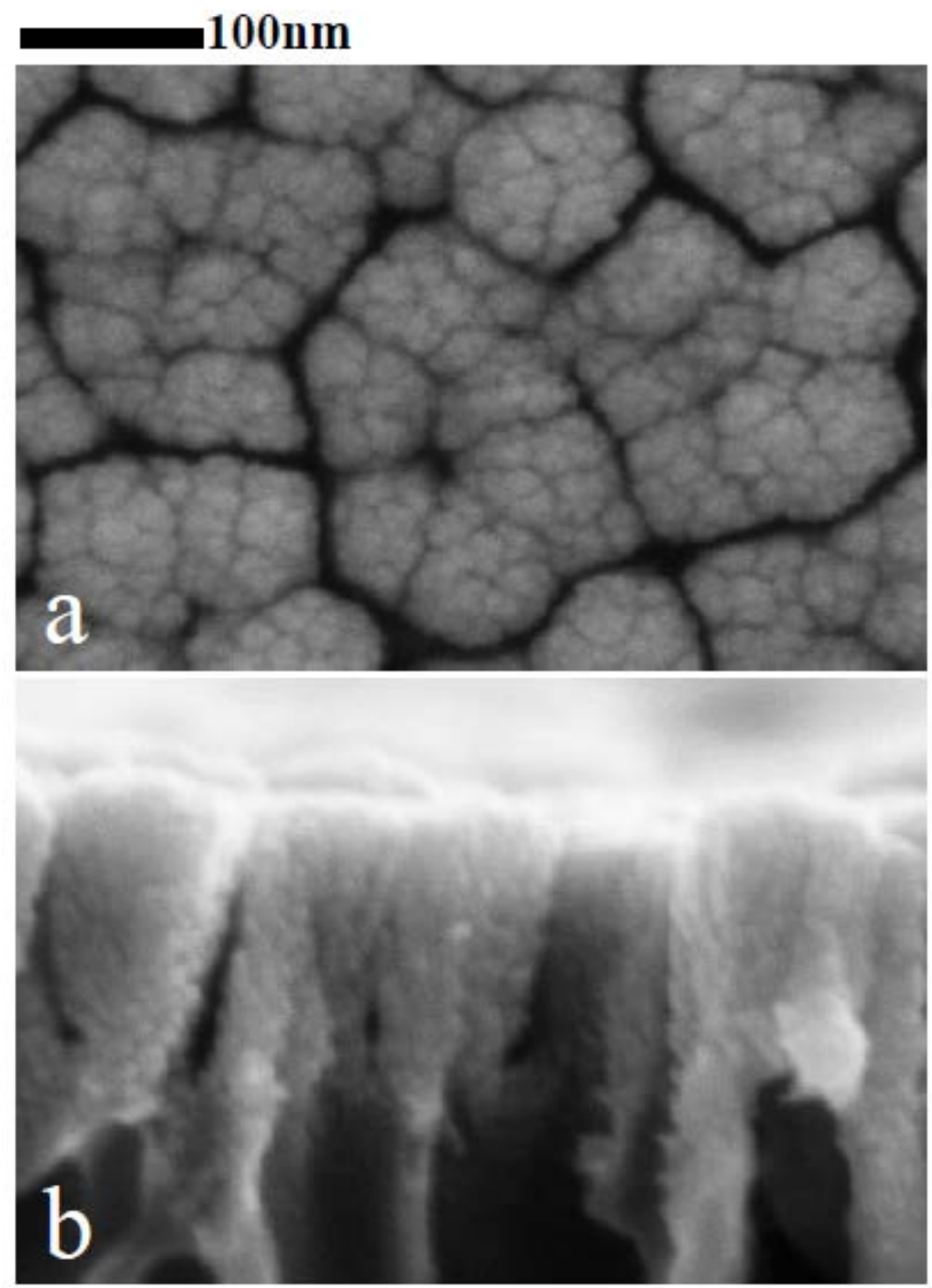

Fig. 1 FE-SEM micrographs of the $\mathrm{Ni}_{80} \mathrm{Fe}_{20}$ film grown on the AAO substrate. (a) Plane view, (b) Cross-sectional view. 
Figure 1 shows FE-SEM micrographs of the $\mathrm{Ni}_{80} \mathrm{Fe}_{20}$ film grown on the AAO substrate. As shown in Fig. 1, the film have a network structure. The network structure is formed by columnar granules connecting with each other. The granule consists of many fine granular grains. The granule size is around $100 \mathrm{~nm}$ and the grain size is about $15 \mathrm{~nm}$. The columnar granules grow on the pore wall of the substrate surface. The granule size is larger than the pore wall width on the substrate surface. The transverse width of the columnar granule gradually widens along the film growing direction. The FE-SEM results are consistent with those reported by references 6 and 7. Figure 2 shows FE-SEM micrographs of the $\mathrm{Ni}_{80} \mathrm{Fe}_{20}$ film grown on the $\mathrm{SiO}_{2} / \mathrm{Si}(100)$ substrate. As shown in Fig. 2, the film is continuous and grows with thin columnar grains. The columnar grain size of the continuous film is almost equal to the granular grain size of the network film.

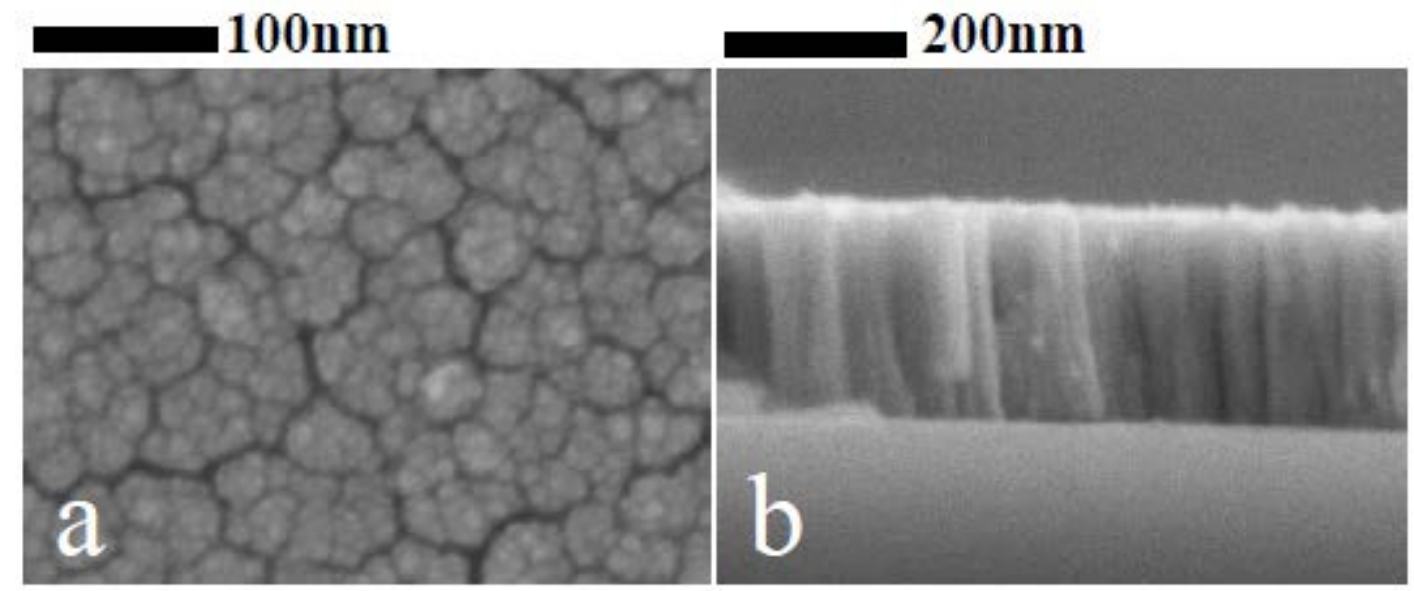

Fig. 2 FE-SEM micrographs of the $\mathrm{Ni}_{80} \mathrm{Fe}_{20}$ film grown on the $\mathrm{SiO}_{2} / \mathrm{Si}(100)$ substrate. (a) Plane view, (b) Cross-sectional view.

As it is well known, shadowing effect can lead to thin films growing with a columnar grain structure. In the present work, the $100 \mathrm{~nm}$ diameter pores of the AAO substrate increase a space between the growing $\mathrm{Ni}_{80} \mathrm{Fe}_{20}$ granules, weakening the shadowing effect during granule growing. The weak shadowing effect could cause that the granule growth rate was almost same in all the directions. The shadow effect on the granule growth was weak at the initial stage of deposition. As the deposition time increased, the granules grew and the space between the granules became small, enhancing the shadowing effect on the granule growth. As a result, the granules exhibit a column-like shape. Then the $\mathrm{Ni}_{80} \mathrm{Fe}_{20}$ network film has the columnar granule structure.

Figure 3 shows a temperature dependence of the resistance (R-T) of the $\mathrm{Ni}_{80} \mathrm{Fe}_{20}$ films grown on $\mathrm{AAO}$ and $\mathrm{SiO}_{2} / \mathrm{Si}(100)$ substrates. In Fig. 3, the resistance $\mathrm{R}$ at each temperature is normalized to the resistance $\mathrm{R}_{3}$ at $3 \mathrm{~K}$. As shown in Fig. 3, the network film grown on the AAO substrate exhibits a minimal value at $35 \mathrm{~K}$ while the continuous film grown on the $\mathrm{SiO}_{2} / \mathrm{Si}(100)$ substrate does not show this minimum. For both the films, a good linear dependence having a linear correlation coefficient of 0.997 is shown in $150-300 \mathrm{~K}$, i.e., a normal R-T relationship of the metallic materials. The temperature coefficient of resistance (TCR) is calculated in terms of the slope of the fitted straight line. The TCR value is $6.1 \times 10^{-4} \mathrm{~K}^{-1}$ for the network film and is $9.4 \times 10^{-4} \mathrm{~K}^{-1}$ for the continuous film. The TCR value of the network film is smaller than that of the continuous film.

As it is well known, if the effect of electron scattering at the film surfaces is negligible, the TCR $\eta$ of the continuous metallic film is given by

$$
\eta=\frac{\rho_{0} \times \eta_{0}}{\rho_{0}+\rho_{\mathrm{i}}+\rho_{\mathrm{g}}}
$$

where $\rho_{0}$ is the resistivity of bulk material at a reference temperature $T_{0}$. $\eta_{0}$ is the TCR of bulk material, also referred to $\mathrm{T}_{0} \cdot \rho_{\mathrm{i}}$ and $\rho_{\mathrm{g}}$ are the temperature-independent resistivities caused by 
impurities and grain boundaries in the film, respectively. On the other hand, for the conductive network film, its conducting mechanism can be explained by the percolation theory. The resistivity $\rho_{\mathrm{f}}$ of the conductive network film is expressed as [9]

$$
\rho_{f}=\left(\rho_{0}+\rho_{i}+\rho_{g}\right) \times\left(V-V_{0}\right)^{-t}
$$

where $\mathrm{t}$ is a positive critical exponent. $\mathrm{V}_{0}$ is the percolation threshold forming the electrical conducting paths in insulating atmosphere. $\mathrm{V}$ is the film volume percentage exceeding $\mathrm{V}_{0}$. The $\eta$ of the conductive network film can be expressed as

$$
\eta=\frac{\rho_{0} \times \eta_{0}}{\rho_{0}+\rho_{\mathrm{i}}+\rho_{\mathrm{g}}} \times\left(V-V_{0}\right)^{t}
$$

In the present work, it is considered that the $\rho_{\mathrm{i}}$ is the same for all the $\mathrm{Ni}_{80} \mathrm{Fe}_{20}$ films because the background pressure, the Ar gas pressure and the deposition rate are the same during the deposition. The continuous film has the thin columnar grain structure while the network film consists of the fine granular grains. The latter has a large amount of grain boundaries compared with the former. Therefore, $\rho_{\mathrm{g}}$ of the network film is larger than that of the continuous film. Furthermore, $\left(\mathrm{V}-\mathrm{V}_{0}\right)^{\mathrm{t}}<1$. According to Eqs. 1 and 3, the TCR of the network film is smaller than that of the continuous film.

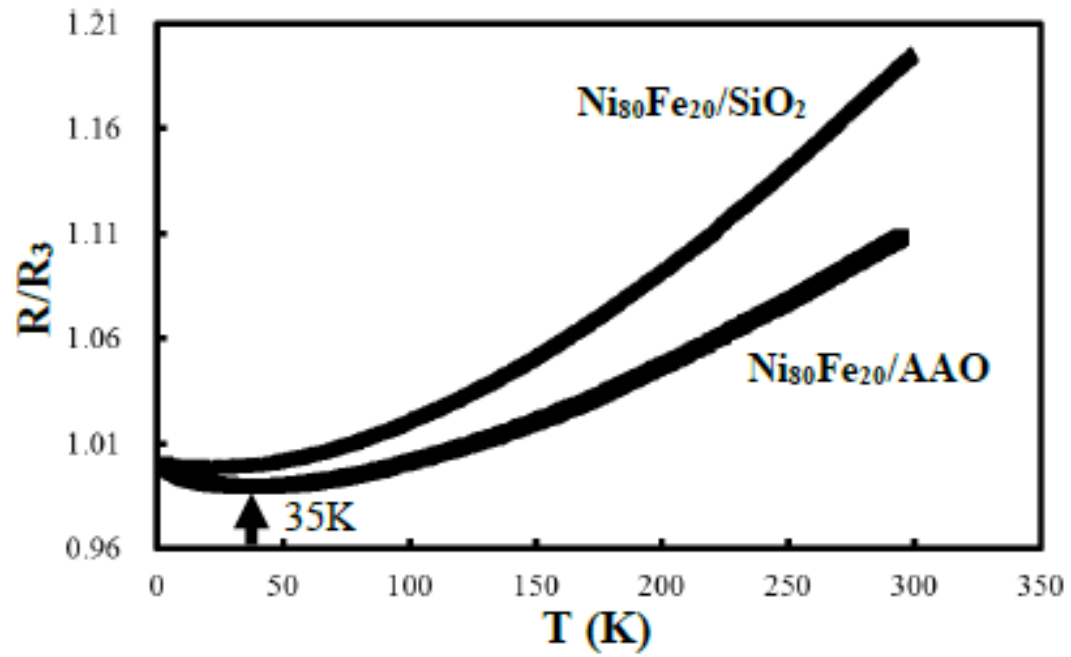

Fig. 3 Temperature dependence of the resistance of the $\mathrm{Ni}_{80} \mathrm{Fe}_{20}$ films grown on $\mathrm{AAO}$ and $\mathrm{SiO}_{2} / \mathrm{Si}(100)$ substrates.

An anomalous temperature dependence of the resistance in two-dimensional metallic films at low temperatures might be explained by the weak localization of conductive electrons and/or the Coulomb electron-electron interaction [5,10-14]. The conductance correction $\Delta \mathrm{G}_{\mathrm{WL}}$ due to the weak localization is expressed as $[10,11,14]$

$$
\Delta \mathrm{G}_{\mathrm{WL}}=\alpha \times \mathrm{p} \times \frac{\mathrm{e}^{2}}{\pi \times \mathrm{h}} \times \ln (\mathrm{T})
$$

where $\mathrm{p}$ is the exponent related to the temperature dependence of the inelastic scattering time of the conductive electrons. $\alpha$ is a constant related to the spin-orbit scattering of the conductive electrons. $T$ is a temperature. $\mathrm{h}$ is the Planck constant and e is the elementary charge. On the other hand, the conductance correction $\Delta \mathrm{G}_{\mathrm{ee}}$ due to the Coulomb electron-electron interaction is given by the expression $[10-12,15]$

$$
\Delta G_{\text {ee }}=(1-\mathrm{F}) \times \frac{\mathrm{e}^{2}}{\pi \times \mathrm{h}} \times \ln (\mathrm{T})
$$


where $\mathrm{F}$ is the three-dimensional electron screening integral factor, $0<\mathrm{F}<1[13,16]$. Actually, $\Delta \mathrm{G}_{\mathrm{WL}}$ and $\Delta \mathrm{G}_{\mathrm{ee}}$ are referred to as the conductance correction $\Delta \mathrm{G}=\mathrm{G}(\mathrm{T})-\mathrm{G}_{0}, \mathrm{G}_{0}$ is a conductance at a reference temperature, $\mathrm{G}(\mathrm{T})$ is a conductance at a certain temperature T. Therefore, according to Eqs. 4 and 5, the conductance of the metallic film at low temperature varies with logarithm of the temperature.

Figure 4 shows a temperature dependence of the conductance $G$ in the low temperature range for the $\mathrm{Ni}_{80} \mathrm{Fe}_{20}$ network film, plotted as $\mathrm{G}$ versus $\ln (\mathrm{T})$. As shown in Fig. 4, a good linear dependence having a linear correlation coefficient of 0.997 is exhibited in 3-30 K. The slope of the fitted straight line is $6.1 \times 10^{-5}$. Then, the prefactor of $\left[\mathrm{e}^{2} /(\pi \times \mathrm{h})\right] \times \ln (\mathrm{T})$ equals to 4.96 . Using Eq. $5, \mathrm{~F}$ is equal to -3.96. This $F$ value is unreasonable because of $0<F<1[13,16]$. Therefore, the Coulomb electron-electron interaction does not dominate the electrical transport property of the $\mathrm{Ni}_{80} \mathrm{Fe}_{20}$ network film at low temperature. On the other hand, using Eq. 4, $\alpha \times$ p equals to 4.96, which might be reasonable value $[10,11]$. Therefore, it is considered that the weak localization of the conductive electrons dominates the electrical transport mechanism in the $\mathrm{Ni}_{80} \mathrm{Fe}_{20}$ network film at low temperature. The 200 nm-thick $\mathrm{Ni}_{80} \mathrm{Fe}_{20}$ network film is rather of three dimensions than of two dimensions. Then a possible explanation could be that the grain boundary structure of the network film forms a quasi-two dimensional arrangement on the surface of the AAO substrate, controlling its electrical transport mechanism.

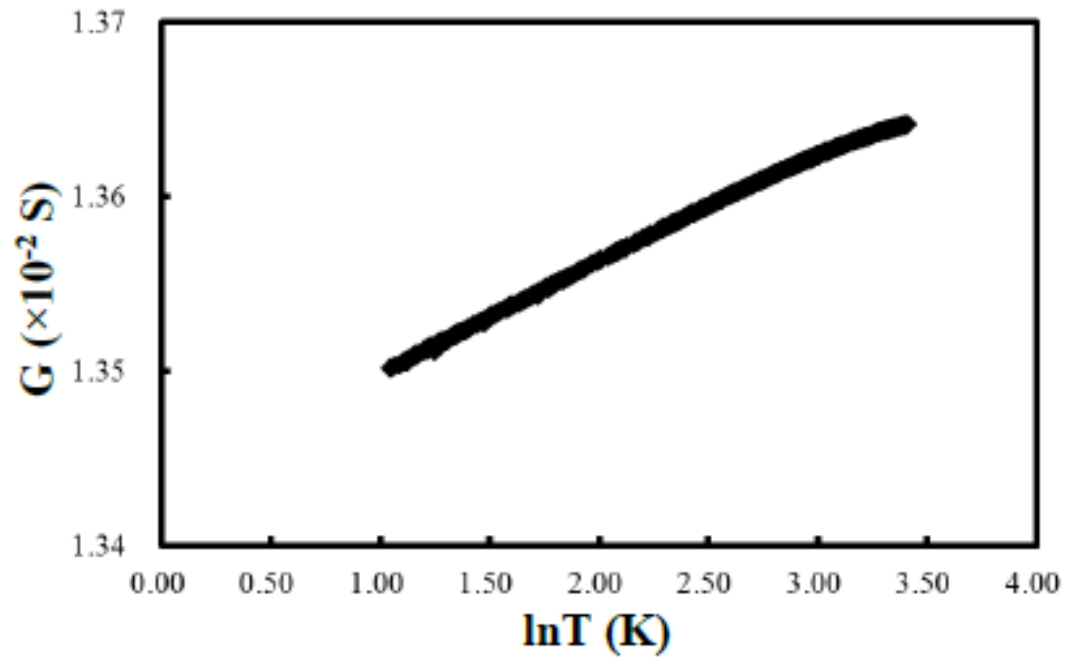

Fig. 4 Temperature dependence of the conductance $\mathrm{G}$ in the low temperature range for the $\mathrm{Ni}_{80} \mathrm{Fe}_{20}$ network film, plotted as $G$ versus $\ln (T)$.

For the electrical transport property of the network film, the result of the present work is well consistent with the results of the $\mathrm{Ni}$ and Fe network films previously reported [6,7]. The electrical transport property at low temperature exhibits the minimal resistance in these network films. The logarithmic R-T relationship is verified at temperatures below $40 \mathrm{~K}$. The two-dimensional weak localization of the conductive electrons dominates the electrical transport mechanism in the network films at low temperature although the network films have a thick film thickness.

\section{Summary}

The $\mathrm{Ni}_{80} \mathrm{Fe}_{20}$ film grown on the AAO substrate has the network structure while that deposited on the $\mathrm{SiO}_{2} / \mathrm{Si}(100)$ substrate is continuous. The network film is formed by columnar granule connection. The columnar granule consists of many fine granular grains. The continuous film grows with thin column grains. The columnar grain size of the continuous film is almost equal to the granular grain size of the network film. The temperature dependence of the resistance within 3-300 K reveals that the network film exhibits the minimal resistance at about $35 \mathrm{~K}$. The logarithmic temperature dependence of the conductance is verified at temperatures below $35 \mathrm{~K}$. The TCR of the network film 
is smaller than that of the continuous film in 150-300 K.

\section{Acknowledgement}

The financial support from the Fundamental Research Funds for the Central Universities No. 8220 is gratefully acknowledged.

\section{References}

[1] J.L. Weston, A. Butera, D. Otte, J.A. Barnard, Nanostructured magnetic networks: a materials comparison, J. Magn. Magn. Mater. 193 (1999) 515-518.

[2] M. Tanaka, E. Saitoh, H. Miyajima, T. Yamaoka, Magnetization process and magnetic structure in a NiFe honeycomb nano-network, J. Magn. Magn. Mater. 282 (2004) 22-24.

[3] L. Sun, Y. Ding, C.L. Chien, P.C. Searson, Exchange coupling in nanostructured CoO/NiFe networks, Phys. Rev. B 64 (2001) 184430.

[4] M. Aprili, P. Nedellec, L. Dumoulin, Study of magnetism in percolating nickel films via extraordinary Hall effect, Solid State Commun. 98 (1996) 221-225.

[5] M. Aprili, J. Lesueur, L. Dumoulin, P. Nedellec, Weak localization and electron-electron interaction in percolating nickel films, Solid State Commun. 102 (1997) 41-46.

[6] H. Zong, H. Qiu, Y.P. Zuo, R.Q. Zheng, J.L. Pan, J.P. He, Structural, electrical and magnetic properties of $\mathrm{Fe}$ network films grown on nanochannel $\mathrm{Al}_{2} \mathrm{O}_{3}$ substrates by direct current magnetron sputtering with the oblique target, Appl. Surf. Sci. 258 (2012) 3237-3243.

[7] G.S. Zou, H. Qiu, Y.P. Zuo, H. Zong, J.P. He, Comparative study on the characteristics of network and continuous Ni films, Thin Solid Films 520 (2012) 6024-6030.

[8] H. Qiu, P. Wu, F.P. Wang, L.Q. Pan, Y. Tian, Structural and electrical properties of Cu films deposited on glass by DC magnetron sputtering, Vacuum 66 (2002) 447-452.

[9] D.I. Tee, M. Mariatti, A. Azizan, C.H. See, K.F. Chong, Effect of silane-based coupling agent on the properties of silver nanoparticles filled epoxy composites, Compos. Sci. Technol. 67 (2007) 2584-2691.

[10] L. van den Dries, C.V. Haesendonck, Y. Bruynseraede, G. Deutscher, Two-dimensional localization in thin copper films, Phys. Rev. Lett. 46 (1981) 565-568.

[11] C.V. Haesendonck, L. van den Dries, Y. Bruynseraede, G. Deutscher, Localization and negative magnetoresistance in thin copper films, Phys. Rev. B 25 (1982) 5090-5096.

[12] G. Bergmann, Quantitative analysis of weak localization in thin Mg films by magnetoresistance measurements, Phys. Rev. B 25 (1982) 2937-2939.

[13] Y. Fehr, S. Maytal, R. Rosenbaum, Important electron-electron interaction and spin-orbit effects in thin copper films, Phys. Rev. B 33 (1986) 6631-6643.

[14] H. White, G. Bergmann, Localization in near-monolayer films, Phys. Rev. B 40 (1989) 11594-11602.

[15] B.L. Altshuler, A.G. Aronov, P.A. Lee, Interaction effects in disorder Fermi systems in two dimensions, Phys. Rev. Lett. 44 (1980) 1288-1291.

[16] T.F. Rosenbaum, R.F. Milligan, G.A. Thomas, P.A. Lee, T.V. Ramakrishnan, R.N. Bhatt, K.DeConde, H. Hess, T. Perry, Low-temperature magnetoresistance of a disordered metal, Phys. Rev.Lett. 47 (1981) 1758-1761. 